If $m_{1}$ and hence $m_{k-1}$ is positive, (9) tells us that all the $m_{k-1}$ members of $S: e_{s_{k-1}+1}, \cdots, e_{s_{k-1}+m_{k-1}}$ belong to $C$. In particular the last one which is $e_{s_{k}}$ belongs to $C$. Moreover $m_{k}=n-\theta\left(s_{k}\right)=0$. Hence $e_{s_{k}}$ is the $n$th member of $C$. If $m_{1}$ and hence $m_{k-1}$ is negative, (9) tells us that all the $m_{k-1}$ members of $S: e_{s_{k+1}}, \cdots, e_{s_{k-1}}$ belong to $C$. In particular $e_{s_{k-1}}$ is a member of $C$. But $m_{k-1}=n-\theta\left(s_{k-1}\right)$ or $\theta\left(s_{k-1}\right)=n-m_{k-1}$; hence $e_{s_{k-1}}$ is the $\left(n-m_{k-1}\right)$ st member of $C$. Thus the proof is complete.

If $S$ and $C$ are wholly arbitrary one may show by means of examples that the conclusion of the theorem is the best that can be obtained from the hypothesis. Additions to the hypothesis make the last two statements of the theorem more precise. For example, if we assume that no two consecutive members of $C$ are consecutive members of $S$, it is easy to prove that $e_{s_{k-1}}$ is the $(n-1)$ st member of $C$ in case $m_{1}<0$.

LEHIGH UNIVERSITY

\title{
A NEW CLASS OF FUNCTIONS OF TWO VARIABLES INVOLVING BESSEL FUNCTIONS OF HALF AN ODD INTEGER*
}

\author{
BY N. A. HALL
}

The evaluation of certain integrals arising in the theory of the conduction of heat between two media of different conductivities $\dagger$ suggested the consideration of the expansions:

$$
\begin{aligned}
\sin \left[\alpha\left(1+x^{2}\right)^{1 / 2}-\beta x\right] & =\sum_{n=0}^{\infty} x^{n} S_{n}(\alpha, \beta), \\
\cos \left[\alpha\left(1+x^{2}\right)^{1 / 2}-\beta x\right] & =\sum_{n=0}^{\infty} x^{n} C_{n}(\alpha, \beta), \\
\exp i\left[\alpha\left(1+x^{2}\right)^{1 / 2}-\beta x\right] & =\sum_{n=0}^{\infty} x^{n} E_{n}^{(1)}(\alpha, \beta), \\
\exp -i\left[\alpha\left(1+x^{2}\right)^{1 / 2}-\beta x\right] & =\sum_{n=0}^{\infty} x^{n} E_{n}^{(2)}(\alpha, \beta) .
\end{aligned}
$$

* Presented to the Society, November 30, 1935.

† G. Green, Philosophical Magazine, (7), vol. 18 (1934), p. 631. 
The four sets of functions appearing in these series are expressible as polynomials in the Bessel functions of half an odd integer and have a number of fairly significant properties.

As is evident from the nature of the generating functions all four sets satisfy the following equations:

$$
\begin{aligned}
& \frac{\partial^{2} u_{n}}{\partial \alpha^{2}}-\frac{\partial^{2} u_{n}}{\partial \beta^{2}}+u_{n}=0, \\
& \frac{\partial^{2} u_{n}}{\partial \beta^{2}}+u_{n-2}=0, \\
& \frac{\partial^{2} u_{n}}{\partial \alpha^{2}}+u_{n}+u_{n-2}=0,
\end{aligned}
$$

together with the special equations

$$
\begin{aligned}
\frac{\partial}{\partial \beta} C_{n}(\alpha, \beta) & =S_{n-1}(\alpha, \beta), \\
\frac{\partial}{\partial \beta} S_{n}(\alpha, \beta) & =-C_{n-1}(\alpha, \beta),
\end{aligned}
$$

and similar relations between $E_{n}{ }^{(1)}$ and $E_{n}^{(2)}$ obtainable from the above by means of

$$
\begin{aligned}
& E_{n}^{(1)}(\alpha, \beta)=C_{n}(\alpha, \beta)+i S_{n}(\alpha, \beta), \\
& E_{n}^{(2)}(\alpha, \beta)=C_{n}(\alpha, \beta)-i S_{n}(\alpha, \beta) .
\end{aligned}
$$

The equations (5)-(11) together with the known values of the functions for $\alpha=0$ and $n=1$ are sufficient to determine the functions uniquely. Solving these equations, we obtain the results:

$$
\begin{aligned}
& E_{n}^{(1)}(\alpha, \beta)=(-i \beta)^{n}\left(\frac{\alpha \pi}{2}\right)^{1 / 2} \sum_{k=0}^{\leqq n / 2} \sigma_{n, k}\left(\frac{\alpha}{2 \beta^{2}}\right)^{k} H_{k-1 / 2}^{(1)}(\alpha), \\
& E_{n}^{(2)}(\alpha, \beta)=(i \beta)^{n}\left(\frac{\alpha \pi}{2}\right)^{1 / 2} \sum_{k=0}^{\leqq n / 2} \sigma_{n, k}\left(\frac{\alpha}{2 \beta^{2}}\right)^{k} H_{k-1 / 2}^{(2)}(\alpha),
\end{aligned}
$$

where

$$
1 / \sigma_{n, k}=\Gamma(n-2 k+1) \Gamma(k+1),
$$

and where $H_{k}^{(1)}(\alpha)$ and $H_{k}^{(2)}(\alpha)$ are Bessel functions of the third 
kind, together with expressions for $S_{n}$ and $C_{n}$ in terms of Bessel functions of the first and second kind. From the properties of the Bessel functions, we may also obtain expressions of the form

$$
S_{n}(\alpha, \beta)=\beta^{n} \sum_{l=0}^{\leqq n / 2} \sum_{k=0}^{l-1} \frac{\sigma_{n, l} \Gamma(l+k)}{\Gamma(l-k) \Gamma(k+1)}\left(\frac{\alpha}{2 \beta^{2}}\right)^{l}(2 \alpha)^{-k}
$$

and

$$
\sin \left(\alpha-(n+l+k) \frac{\pi}{2}\right),
$$

$$
\begin{aligned}
C_{n}(\alpha, \beta)= & \sin \left(\alpha-n \frac{\pi}{2}\right) \sum_{k=0}^{\leqq n / 2} \sigma_{n, k}\left(\frac{\alpha}{2 \beta^{2}}\right)^{k} R_{k-1,1 / 2}(\alpha) \\
& -\cos \left(\alpha-n \frac{\pi}{2}\right) \sum_{k=0}^{\leqq n / 2} \sigma_{n, k}\left(\frac{\alpha}{2 \beta^{2}}\right)^{k} R_{k-2,3 / 2}(\alpha),
\end{aligned}
$$

where $R_{m, n}(\alpha)$ is the Lommel polynomial.*

From the well known infinite integrals involving the Bessel functions, a number of polynomials may be expressed in terms of infinite integrals involving these new functions. Among the most interesting are the expressions for the truncated hyperbolic sine and cosine series:

$$
\begin{aligned}
& \sum_{\tau=0}^{n} \frac{\beta^{2 \tau+1}}{(2 \tau+1) !} \\
& =(-)^{n+1} \frac{\cosh ^{2 n+1} \tau}{\sinh \tau} \int_{0}^{\infty} e^{-\alpha \sinh \tau} S_{2 n+1}(\alpha, \beta \operatorname{sech} \tau) d \alpha, \\
& \sum_{\tau=0}^{n} \frac{\beta^{2 \tau}}{(2 \tau) !}=(-)^{n} \frac{\cosh ^{2 n} \tau}{\sinh \tau} \int_{0}^{\infty} e^{-\alpha \sinh \tau} C_{2 n}(\alpha, \beta \operatorname{sech} \tau) d \alpha .
\end{aligned}
$$

Of a more complicated nature are

$$
\int_{0}^{\infty} E_{n}^{(1)}(\alpha, \beta) d \alpha=(-i)^{n+1} \frac{\beta^{n}}{n !}{ }_{3} F_{0}\left(-\frac{n}{2},-\frac{n-1}{2}, \frac{1}{2} ; \frac{4}{\beta^{2}}\right),
$$

and

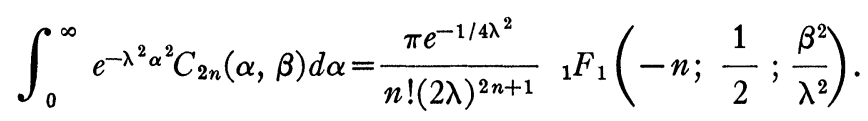

In addition to the above series expansions for the functions,

* G. A. Watson, Theory of Bessel Functions, p. 296. 
we may obtain from them or direct from the generating functions expressions in terms of contour integrals. Making use of the contour integrals for the Bessel functions* we obtain results of the type:

$$
E_{n}^{(2)}(\alpha, \beta)=\frac{(i)^{n}}{(\pi)^{1 / 2}} \int_{C} e^{\alpha^{2} / \tau^{2}-\tau^{2} / 4} \chi_{n}\left(\beta,-\frac{\alpha^{2}}{\tau^{2}}\right) d \tau,
$$

where the contour starts at the origin in the direction of the positive imaginary axis, bends around and extends to infinity along the positive real axis; and where $\chi_{n}(s, t)$ is a polynomial related to the Hermite polynomial, $\uparrow$

$$
\begin{aligned}
\chi_{n}(s, t) & =\frac{(2 t)^{n}}{n !} e^{-s^{2} /(4 \nu)} \frac{\partial^{n}}{\partial s^{n}}\left(e^{s^{2} /(4 \nu)}\right)=s^{n} \sum_{m=0}^{\leqq n / 2} \sigma_{n, m}\left(\frac{t}{s^{2}}\right)^{m} \\
& =\frac{(-t)^{n / 2}}{n !} H e_{n}\left(\frac{s}{2 i t^{1 / 2}}\right) .
\end{aligned}
$$

A similar expression exists for $E^{(1)}(\alpha, \beta)$, where in (20) $i$ is replaced by $-i$ and the contour is the same but below the positive real axis.

In conclusion it might be stated that on setting $\beta=0$ the expansions (1)-(4) become simply special cases of Lommel's expansion of a Bessel function with an irrational variable. $\ddagger$ An extension of these expansions to obtain generalizations of our functions of two variables might be of some interest, although it introduces hypergeometric series of the type ${ }_{2} F_{3}$.

California Institute of Technology

* Watson, loc. cit., p. 178.

$\dagger$ H. Lacrose, Comptes Rendus, vol. 148 (1909), pp. 770-773.

$\ddagger$ Watson, loc. cit., p. 140. 